\title{
New Algorithms for Beam Formation and its Comparison
}

\author{
Kishore M \\ Department of Electronics and \\ Communication \\ PDIT, Hospet, India
}

\author{
H M Guruprasad \\ Department of Electronics and \\ Communication \\ PDIT, Hospet, India
}

\author{
Ramesh K \\ Department of Computer \\ science Engineering \\ Karnataka University \\ Dharwad, India
}

\begin{abstract}
Interference reduction is vital for being able to effectively communicate with mobile users. In order to provide line of sight communications and continual coverage to the remote users. One approach to increasing capacity and coverage zones for the servicing wireless station is to use smart antennas. Sophisticated adaptive beamforming techniques can be applied to point the array's beam in the desired look direction while simultaneously nulling out the interfering signal. This paper explains the beam formation by using Signum Data-LMS and compared the performance with LMS and LLMS algorithm.
\end{abstract}

\section{Keywords}

Adaptive Array, Smart Antenna, Beamforming.

\section{INTRODUCTION}

In recent years a substantial increase in the development of broadband wireless access technologies for evolving wireless internet services and improved cellular systems has been observed. It is widely foreseen that in the future an enormous rise in traffic will be experienced for mobile and personal communication systems. This is due to an increase in number of users and introduction of new high bit rate data services. This trend is observed for second-generation systems as well and it will most certainly continue for third-generation systems. The rise in traffic will put a demand on both manufacturers and operators to provide sufficient capacity in the network, this becomes a major challenging problem for the service providers to solve. There are certain negative factors like co-channel interference and fading in the radiation environment contributing to the limit in the capacity. Smart antenna is one such development in this direction to full fill the feature requirements of mobile networks.

\section{LITERATURE SURVEY}

Multipath propagation, defined as the creation of multiple signal paths between the transmitter and the receiver due to the reflection of the transmitted signal by physical obstacles is one of the major problems of mobile communications It is well known that the delay spread and resulting intersymbol interference (ISI) due to multiple signal paths arriving at the receiver at different times have a critical impact on communication link quality. On the other hand, co-channel interference is a major limiting factor on the capacity of wireless systems, resulting from the reuse of the available network resources by a number of users. Smart antenna systems can improve link quality by combating the effects of multipath propagation or constructively exploiting the different paths, and increase capacity by mitigating interference and allowing transmission of different data streams from different antennas.

\section{SMART ANTENNA}

The smart antenna consists of Down Converter (D/C), Analog to Digital (ADC) converters and a processor in which which DOA and Adaptive Beamforming (ABF) algorithms are present as shown infig1.It also consists of a uniform linear antenna array for which the current amplitudes are adjusted by a set of complex weights using an adaptive beamforming algorithm. The adaptive beamforming algorithm optimizes the array output beam pattern such that maximum radiated power is produced in the directions of desired mobile users and deep nulls are generated in the directions of undesired signals representing co-channel interference from mobile users in adjacent cells. Prior to adaptive beamforming, direction-of arrival estimation algorithm.

\section{BEAMFORMING}

A Beamformer is a set of sensors (antennas) arranged in a linear fashion with outputs that can be steered electronically. The sensor array comprises of isotropic antenna elements that extract spatial information from the waves emitted by signal sources. The signal received at these sensors is sent for computation of weights. One can define beamforming as adjusting the sensitivity of antenna in a certain specified frequency. So, in essence, beamforming is spatio-temporal filtering where signals are separated according to their directional characteristics and frequency content.

\subsection{Adaptive beamforming:}

Adaptive Beamforming is a technique in which an array of antennas is exploited to achieve maximum reception in a specified direction while rejecting signals of the same/different frequency from other directions. The weights are computed and adaptively updated in real time based on signal samples. The adaptive process permits narrower beams in look direction and reduced output in other directions, which results in significant improvement in Signal to Interference Noise Ratio (SINR).

\subsection{COMPLEX WEIGHTS.}

The weight update equation is given by $w(n+1)=w(n)+\Delta w(n)$

Where, $\Delta w(n)$ is the correction applied to calculate new weights. This type of weight updating for $w(n)$ forms the heart of every beamforming algorithm and each of the beamforming algorithms varies in terms of computation of weights.In a practical case, if $s(n)$ is the signal samples corresponding to look direction, $\mathrm{i}(\mathrm{n})$ is interfering signal samples corresponding to jamming directions and $n_{0}(n)$ is noisy signal samples due to receiver components. The induced signal is given by 
$x(n)=s(n) a\left(\theta_{0}\right)+\sum_{i=1}^{M} i_{i}(n) a\left(\theta_{i}\right)+n_{0}(n)$

Where, $\mathrm{M}$ is number of jamming sources, $a\left(\theta_{0}\right)$ is desired steering vector and $a\left(\theta_{i}\right)$ is the steering vector corresponding to $i^{\text {th }}$ interference signal.Since jamming signals (or interfering signals) are of no interest, it is assumed $i(n)_{1}=i(n)_{2} \ldots \ldots=i(n)_{M}=i(n) \quad$ with this modification equation (1) can be written as

$$
x(n)=a\left(\theta_{0}\right) s(n)+i(n) \sum_{i=1}^{M} a\left(\theta_{i}\right)+n_{0}(n)
$$

In matrix notation, induced signal can be written as

$$
X=A_{\theta_{0}} S+A_{i n} I_{i}+N
$$

Where,$X$ represents $\mathrm{L} \times \mathrm{N}_{\mathrm{s}}$ induced signal matrix, $\mathrm{N}_{\mathrm{s}}$ is total number of samples, ' $L$ ' represents number of array elements, ' $S$ ' represents reference signal samples. $A_{\theta_{0}}$ is desired steering vector of order Lx1, $\boldsymbol{I}_{i}$ represents interference signal samples matrix of order $1 \mathrm{xN}_{\mathrm{s}}, N$ represents Gaussian noise matrix of order $\mathrm{LxN}_{\mathrm{s}}$ and $\boldsymbol{A}_{\boldsymbol{i n}}$ is Lx 1 column vector that is obtained by adding all columns of array manifold vector.

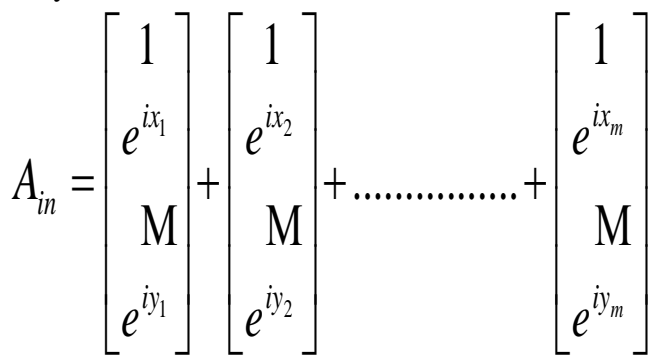

$\mathrm{X} 1=2 \pi \mathrm{d} \sin \theta_{1}, \mathrm{X} 2=2 \pi \mathrm{d} \sin \theta_{2}, \mathrm{Xm}=2 \pi \mathrm{n} \sin \theta_{m}$

$\mathrm{Y} 1=2 \pi \mathrm{d}(\mathrm{L}-1) \sin \theta_{1}, \mathrm{Y} 2=2 \pi \mathrm{d}(\mathrm{L}-1) \sin \theta_{2}$

$\mathrm{Ym}=\mathrm{Ym}=2 \pi \mathrm{d} \sin \theta_{m}$

Where, ' $\mathrm{d}$ ' is the distance between antenna elements, $\theta_{1}, \theta_{2}, \ldots \ldots, \theta m$ are directions of jamming signals and $\mathrm{M}$ is number of jamming signals.

\subsection{Simulation Methodology of ABF.}

1. Compute the Lx1 steering vector for desired direction $\theta_{0}$.

2. Compute the LxM array manifold vector corresponding to $\mathrm{M}$ interference source directions $\theta_{1}, \theta_{2}, \ldots \ldots . . ., \theta_{M}$.
3. Obtain signal samples ' $\mathrm{S}$ ' by sampling continuous time signal of baseband frequency. (For simulation sine wave samples is considered).

4. Compute the autocorrelation matrix $R_{x x}$

5. Compute the step size

6. Compute the following for all signal samples $0 \leq n \leq N_{s}$.

Where, $N_{s}$ is the total number of signal samples.

7. The array factor is computed

8. Array factor versus angles are plotted.

\section{LEAST MEAN SQUARE (LMS)}

The LMS algorithm is the most widely used adaptive beamforming algorithm, being employed in several communication applications. It has gained popularity due to its low computational complexity and proven robustness. The LMS algorithm changes the weight vector $w(n)$ along the direction of the estimated gradient based on the steepest descent method. In employing the LMS algorithm, it is assumed that sufficient knowledge of the reference signal is present.

The Least Mean Square (LMS) algorithm is one of the most popular algorithms in adaptive signal processing, due to its simplicity and robustness. Many different modifications were proposed to improve performance of the LMS and a large number of results on its steady state misadjustment and its tracking ability has been obtained.

\section{LEAKY LEAST MEAN SQUARE (LLMS)}

This is the variation of LMS algorithm The Eigen value decomposition of autocorrelation matrix $\boldsymbol{R}_{x x}$ sometimes produces zero Eigen values, then LMS adaptive filter has one or more modes that are un-driven or un-damped. Since it is possible for these un-damped modes to become unstable, it is important to stabilize the LMS by forcing these modes to zero. One way to accomplish this is to introduce leakage coefficient $\gamma$ into auto correlation matrix, step size and weight vector equation.

\section{SIGNUM DATA-LMS (SDLMS)}

In spite of computational efficiency of LMS and LLMS algorithms, additional simplifications may be necessary in some applications, such as high speed digital communication. The weight coefficient of LMS is modified by applying sign operator to data.

The weight vector for Signum Data Least Mean Square (SDLMS) is given by

$$
w(n+1)=w(n)+\mu \operatorname{sgn}(x(n)) e(n)
$$




\section{SIMULATION RESULTS}

Table 1: Inputs for individual algorithms

\begin{tabular}{|l|l|l|l|}
\hline Algorithm & $\begin{array}{l}\text { Desired } \\
\text { Angle }\end{array}$ & $\begin{array}{l}\text { Jammer } \\
\text { Angle }\end{array}$ & $\begin{array}{l}\text { No Of } \\
\text { Antenna } \\
\text { Elements }\end{array}$ \\
\hline LMS & 45 & 5 & 100 \\
\hline LLMS & 45 & 5 & 100 \\
\hline SE-LMS & 45 & 5 & 100 \\
\hline
\end{tabular}

Table 2: Inputs for Comparison

\begin{tabular}{|l|l|l|l|}
\hline Algorithm & $\begin{array}{l}\text { Desired } \\
\text { Angle }\end{array}$ & $\begin{array}{l}\text { Jammer } \\
\text { Angle }\end{array}$ & $\begin{array}{l}\text { No Of } \\
\text { Antenna } \\
\text { Elements }\end{array}$ \\
\hline LMS & 50 & 5 & 100 \\
\hline LLMS & 60 & 10 & 100 \\
\hline SE-LMS & 70 & 15 & 100 \\
\hline
\end{tabular}

LMS Algorithm

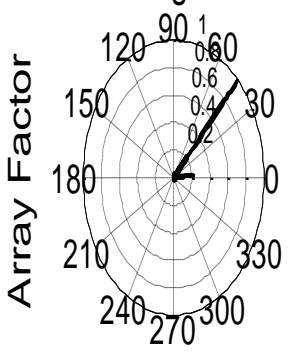

Angle(deg)

Figure 1:Polar plot of LMS algorithm

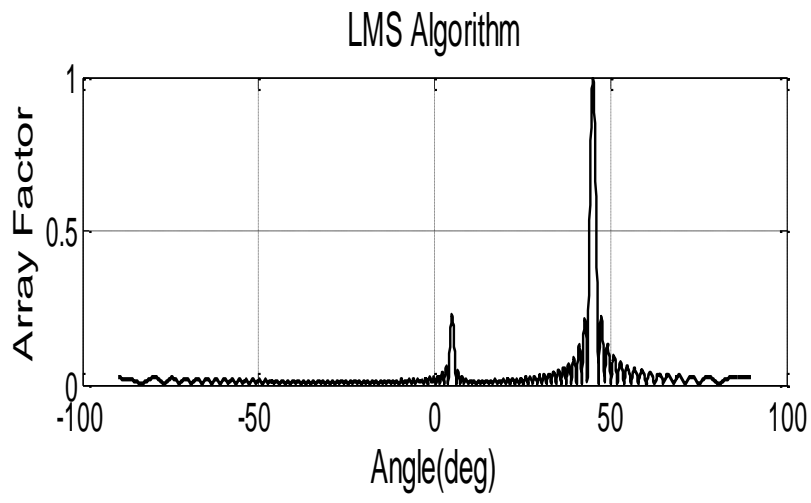

Figure 2:Beam plot of LMS algorithm

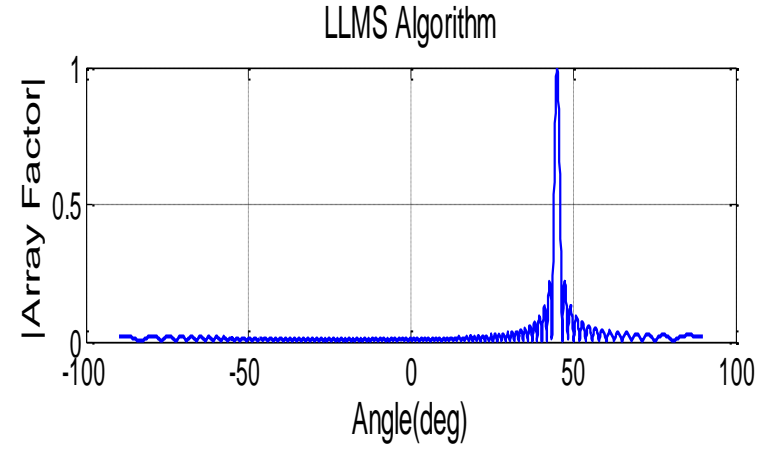

Figure 3:Beam plot of LLMS algorithm

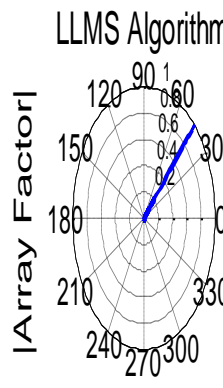

Angle(deg)

Figure 4:Polar plot of LLMS algorithm

SD-LMS Algorithm

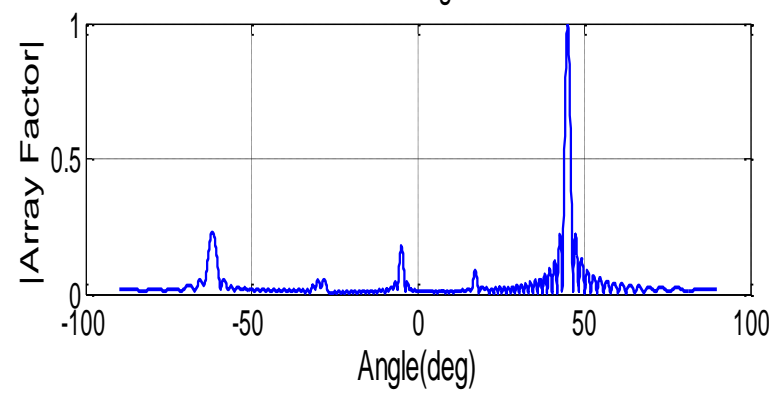

Figure 5:Polar plot of SDLMS algorithm

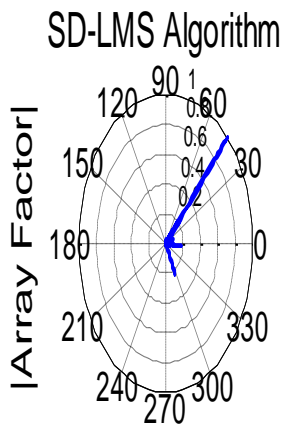

Angle(deg)

Figure 6:Polar plot of SDLMS algorithm 


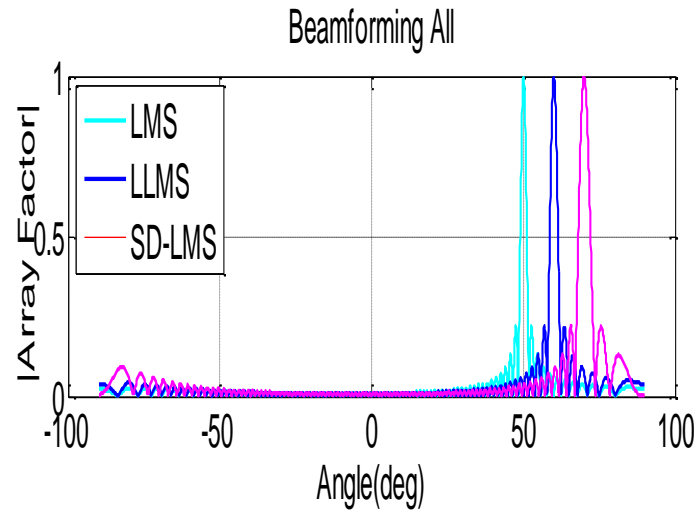

Figure 7:Comparison of Beamforming algorithms

\section{FUTURE SCOPE}

Beamforming is a technology that promises to reshape the future of Telecom industry by focusing on problems of transmission fidelity and spectral efficiency. There have been intensive developments in this field. There is still scope for future improvements. Some of the drawbacks can be overcome by extension of this research work which is listed

1. Uniform linear array of sensors can be replaced with Uniform Circular Array [UCA] of sensors, which increases resolution of beam and removes symmetrical structure about array axis.

2. The Beamforming algorithms simulated in this project took only the present samples in estimating the weight vector but new algorithms use both present as well as past data which increases accuracy. One such algorithm is Quasi Newton Beamforming algorithm.

3. Many applications also require steering of nulls in jammer directions hence Null Steering Beamformer can be implemented to steer nulls.

\section{CONCLUSION}

A new adaptive algorithm for beamforming called signum data least mean square(SDLMS) along with conventional least (LLMS) Algorithm is presented and analyzed. The proposed method works well to form the main beam in the desired user and reduced interference in the undesired direction. When the proposed method is compared with LMS and LLMS algorithm with respect to main beam formation ,The proposed method works well as shown in the figure 7,simulateounsouly producing deep nulls towards the jammers

\section{REFERENCES}

[1] L. C. Godara, "Application of antenna arrays to mobile communication. II. Beam-forming and direction-ofarrival considerations", Proceedings of IEEE, August 1997, Vol-85, Issue 8, pp 1195- 1245.
[2] M. Mouhamadou and P. Vaudon, "Smart Antenna Array Patterns Synthesis: Null Steering and multi-user Beamforming", Progress in Electromagnetic research, June 2006, PIER-60, pp 95-106.

[3] J.M. Samhan, R.M. Shubair and M.A.Al-qutayriz , "Design and implementation of an adaptive smart antenna array system", Innovations in information technology, November 2006, pp 1-4.

[4] Chen sun, Nemai Chandra Karmakar, "Direction of Arrival Estimation based on a Single-Port Smart Antenna using MUSIC Algorithm with periodic signals", International Journal of Signal Processing, March 2005, Vol-1, No 2, pp153-162.

[5] Chen sun and Nemai Chandra Karmakar, "Direction of Arrival Estimation with a Novel single port smart antenna", EURASIP Journal on applied Signal Processing, Sept 2004, Vol-2004, pp 1364-1375.

[6] T. K. Sarkar, S. Park, J. Koh and R.A. Schneible, "A Deterministic Least Squares Approach to Adaptive Antennas", Digital Signal Processing, A Review Journal6, March 1996, Vol- 49 , pp 185-194.

[7] Seungwon Choi, Hong-Min Son, and Tapan K sarkar, "Implementation of a Smart Antenna System on a General-Purpose Digital Signal Processor Utilizing a Linearized CGM", Digital Signal Processing Journal-7, March 1997,Vol- 7, Issue-8,pp 105-119.

\section{AUTHOR'S PROFILE}

Mr. Kishore M, Currently working as a faculty in Proudadhavaraya Institute of Technology (PDIT), Department of Electronics and communication. Completed his master degree in Canara Engineering College, Mangalore, and specialization as an Applied Electronics. Worked as a project engineer in a various industries, Bangalore. His current area of interest includes Antenna theory and design, wireless communication and Networking.

Prof. H M Guruprasad, working in Proudadhavaraya Institute of Technology, Department of Electronics and Communication. His area of interest VLSI, Analog communication.

Prof. Ramesh K, currently working in Karnataka University, Dharwad, Department of Computer Science Engineering, His area of interest in optical fiber communication, protocols and networking. Served as a faculty in various reputed colleges. 